\title{
Ubiquitous Intelligent Sensing System for a Smart Home
}

\author{
Jonghwa Choi, Dongkyoo Shin, and Dongil Shin* \\ Department of Computer Science and Engineering, Sejong University, 98 Kunja-Dong \\ Kwangjin-Gu, Seoul, Korea \\ jhchoi@gce.sejong.ac.kr, shindk@sejong.ac.kr, dshin@sejong.ac.kr
}

\begin{abstract}
We present the ubiquitous intelligent sensing system for a smart home in this paper. A smart home is intelligent space that studies patterns of home contexts that is acquired in a home, and provides automatic home services for the human. The ubiquitous intelligent sensing system acquires seven sensing contexts from four sensor devices. We utilize association rules of data mining and linear support machine to analyze context patterns of seven contexts. Also, we analyze stress rates of the human through the HRV pattern of the ECG. If the human is suffering from stress, the ubiquitous intelligent sensing system provides home service to reduce one's stress. In this paper, we present the architecture and algorithms of the ubiquitous intelligent sensing system. We present the management toolkit to control the ubiquitous intelligent sensing system, and show implementation results of the smart home using the ubiquitous intelligent sensing system.
\end{abstract}

\section{Introduction}

The computer industry has seen wonderful results in miniaturization and performance improvement of devices as a result of rapid technological development: central computing with mainframes (1950s through the 1980s), personal computers (1980s to present), and computer networks (1990s to present) [1]. A fourth era is now emerging as computers became ubiquitous, a technology more noticeable by its absence than its presence [2,3]. This paper addresses the architecture of a ubiquitous intelligent sensing system and algorithms of components that compose the system. A future home is an intelligent space that realizes ubiquitous computing that offer the human a more comfortable life. Ubiquitous computer aims to "enhance" computer use by making many computers available throughout the physical environment, but making them effectively invisible to the user [4]. A smart home helps to provide a more comfortable home life to humans through intelligent sensing that offers a human automated service in a ubiquitous space. Studies of intelligent agents in a smart home have proceeded into many different a lot of directions. MS Easy Living provides a home service through location tracking of the human [5]. MavHome presented a prediction algorithm of home service in smart home, and applied data mining as its algorithm [6, 7].

We implemented four sensor devices (ECG sensor, home temperature sensor, network camera for human location and motion and facial expression sensor) that acquire home contexts from human and home. The sensor devices provide seven

\footnotetext{
Corresponding author.
} 
sensing contexts (ECG, pulse, body temperature, home temperature, human location, human motion and facial expression) to the ubiquitous intelligent sensing system. We apply LSVM (linear support vector machine) and association rules of data mining to analyze patterns of all contexts.

Section 2 gives related research work on an intelligent sensing system. Section 3 addresses architecture of the ubiquitous intelligent sensing system for the smart home. In section 4, we explain a detailed algorithm of the components that compose the intelligent sensing system. Section 5 presents implementation and experimental results. We conclude with section 6 .

\section{Related Studies}

Perry and Dowdall documented the rationale and design of a multimodal interface to a pervasive/ubiquitous computing system that supports independent living by older people in their own homes [8]. The Smart-In-House project used a system of basic sensors to monitor a person's in-home activity; a prototype of the system is being tested within a subject's home [9]. They examined whether the system could be used to detect behavioral patterns and report the results. Alex and Brent discuss the use of computer vision in pervasive healthcare systems, specifically in the design of a sensing agent for an intelligent environment that assists older adults with dementia during the activity of daily living [10]. The 1:1 pro system constructed personal profiles based on the customer's transactional histories. The system used data mining techniques to discover a set of rules describing customer's behavior and supports human experts in validating the rules [11]. Kehagias and Petridis introduced the PREdictive MOdullar Fuzzy System (PREMOFS) to perform a time series classification. A PREMOFS consists of a bank of predictors and a fuzzy inference module. The PREMOFS is a fuzzy modular system that classifies the time series as one of a finite number of classes, using the full set of un-preprocessed past data to perform a recursive, adaptive and competitive computation of membership function, based on predictive power [12].

\section{Architecture of Ubiquitous Intelligent Sensing System}

Figure 1 shows the architecture of the ubiquitous intelligent sensing system for a smart home. Figure 1 presents ten sensing contexts, and we provide the intelligent sensing system with seven sensing contexts and time for pattern prediction of the home service. Eye tracker and voice sensing are processed by a rule-based algorithm.

Five home appliances have been connected to the wireless network in the laboratory. The ubiquitous intelligent sensing system predicts a home service pattern of the human, and offers automatic home service for the human. We use the SAPR (supervised algorithm for pattern recognition: a linear support vector machine) and the data miner (association rules) to analyze the home service for the human. The ubiquitous intelligent sensing system studies the human's home service pattern without providing a home service for the human during the learning phase. For example, when the human turns on the TV, the context extractor acquires the human's home service command (TV-On) and sensing contexts from four sensor devices, and transmits all data 
to the pattern recognition process. During the prediction phase of the home service, the ubiquitous intelligent sensing system acquires seven sensing contexts and the time from the sensor devices every three seconds, and it provides an automatic home service to the human through pattern analysis of previous home services. The context extractor acquires all contexts from sensor devices, and manages them. The context extractor processes contexts in two steps to analyze the pattern of eight contexts acquired from sensor devices. First, the context extractor normalizes eight contexts between 0.1 and 0.9 so that contexts are treated by input value (train input and test input) of the supervised algorithm for pattern recognition. Second, the context extractor stores all contexts in the database for creation of association rules. We applied the HHIML that is based on XML as the context's management structure. This helped to easily approach all contexts in other components, and presents an efficient interface to the web $[13,14]$. The home service provider executes home service that is predicted from the supervised algorithm for pattern recognition and association rules of data mining.

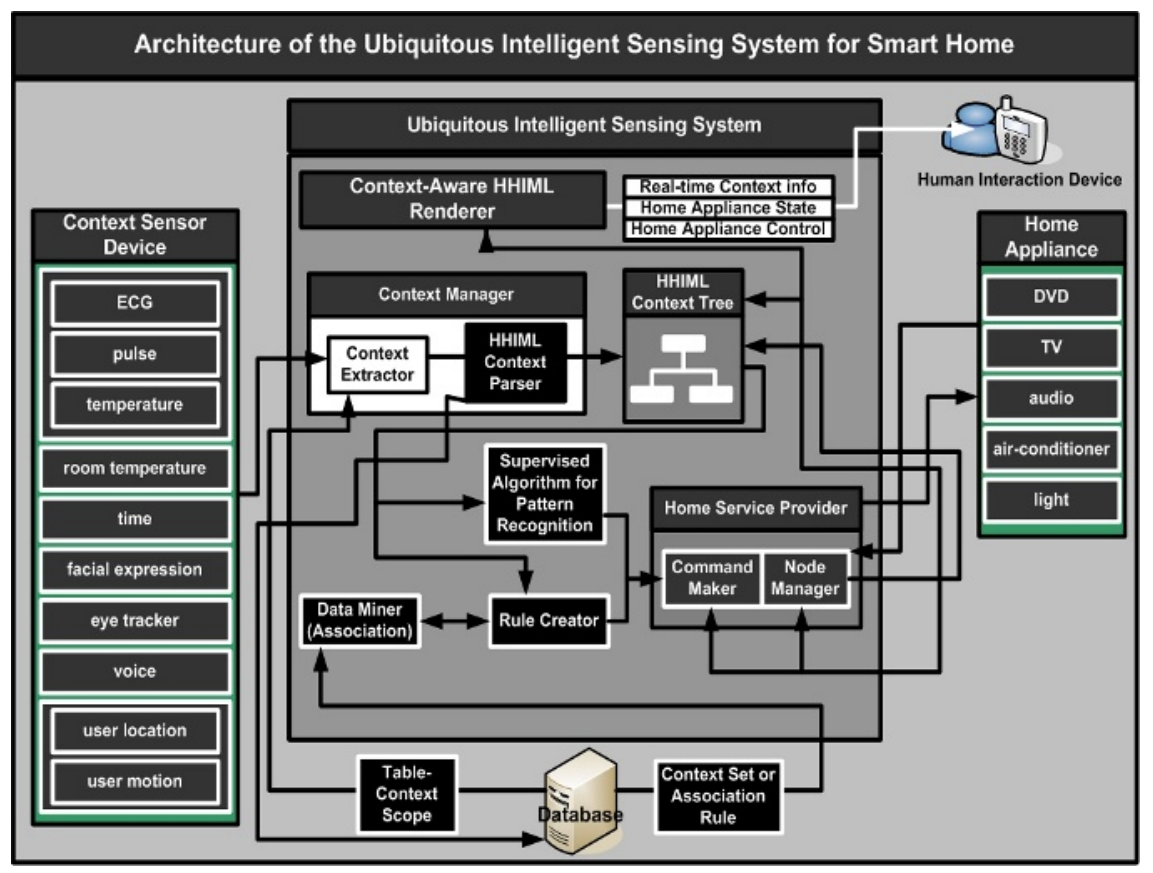

Fig. 1. Architecture of the ubiquitous intelligent sensing system for a smart home

\section{Components of the Ubiquitous Intelligent Sensing System}

\subsection{Context Extraction and Processing}

Figure 2 shows pictures of four sensor devices used to acquire seven contexts for the pattern recognition of home service. 


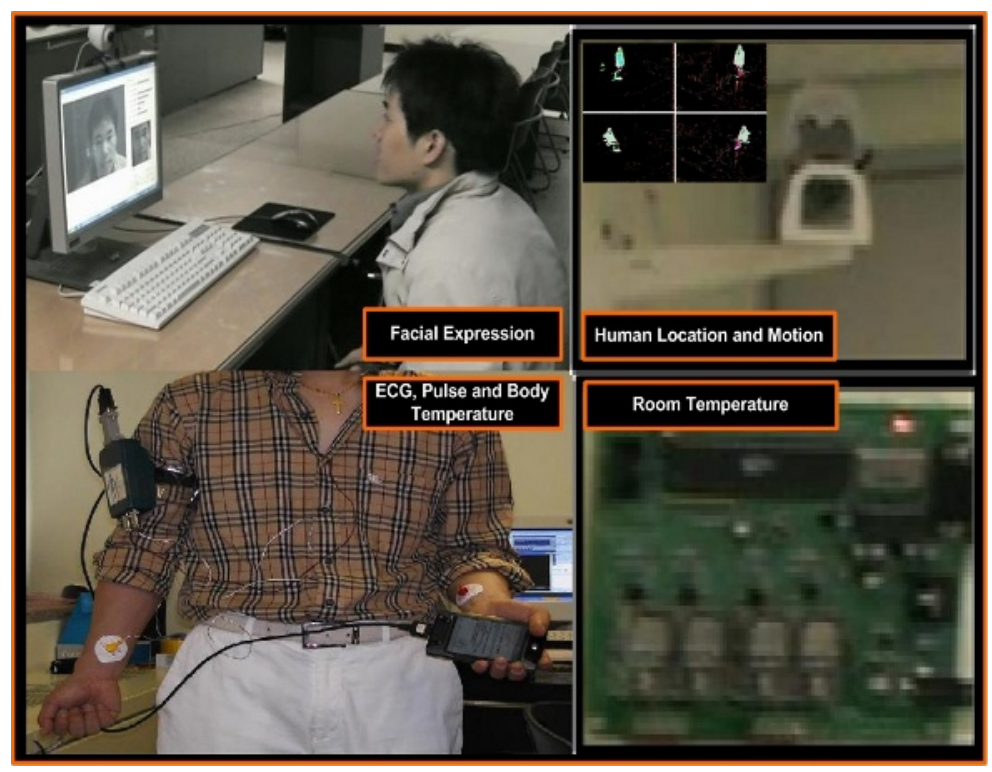

Fig. 2. Four sensor devices for extraction of seven contexts

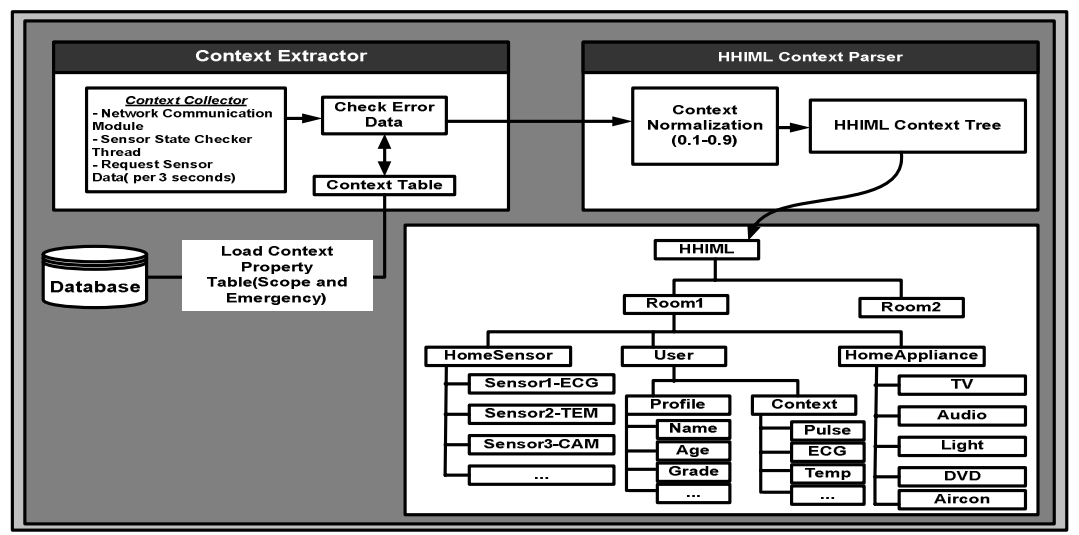

Fig. 3. Structure of the context extractor that takes charge of the sensing context's extraction and context processing

The four sensor devices use a WiFi for network communication with the ubiquitous intelligent sensing system. The facial expression sensor recognizes the human's expression by comparing it with seven standard expressions (blank, surprise, fear, sad, angry, disgust and happy). They are categorized as described in [15]. The human location is decided from absolute coordinates through analysis of raw images. The human's motion is recognize from six motions (lie, stand, sit, sit_gesture1, sit_gesture2 and sit_gesture3) using pattern recognition algorithms. 
We acquire the ECG signal, pulse and temperature from the ECG sensor device. The pulse and the temperature are transmitted to the pattern recognition algorithm for pattern analysis of the home service: the ECG signal is used to predict human's stress. Figure 3 shows the structure of the context extractor that takes charge of sensing context's extraction and context processing.

The context extractor acquires contexts from the sensor devices that is presented in figure 2, and normalizes all contexts between 0.1 and 0.9 . Then, it converts all contexts that are acquired in real-time into the HHIML's tree that is based on XML to manage all contexts efficiently.

\subsection{Pattern Recognition of Human's Home Service}

Figure 4 shows the structure of the pattern recognition algorithm that predicts the home service that the human wants.

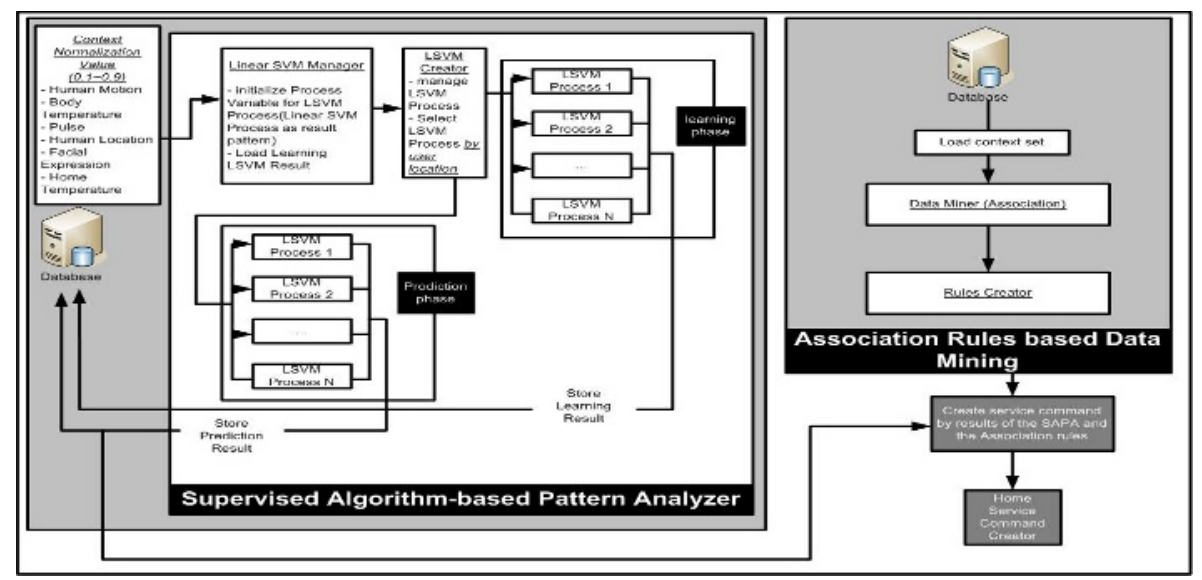

Fig. 4. Structure of the supervised algorithm-based pattern analyzer and the association rules based data mining

We applied LSVM by hierarchal structure for pattern recognition of three home services (TV, audio and DVD). The ubiquitous intelligent sensing system measures stress of a human from analysis of the ECG. If the human is suffering from stress, the ubiquitous intelligent sensing system provides a home service (soft music and a faint light) to comfort the human and reduce stress. The ECG (electrocardiogram) is an electric signal, which reflects the hearts pulse rate that is measurable on the body's surface. Figure 5 shows the ECG graph with P, Q, R, S and T values that were extracted from an ECG signal.

The ubiquitous intelligent sensing system provides a home service that reduces the stress of a human using the HRV (Heart Rate Variability) of the ECG signal $[16,17]$. 


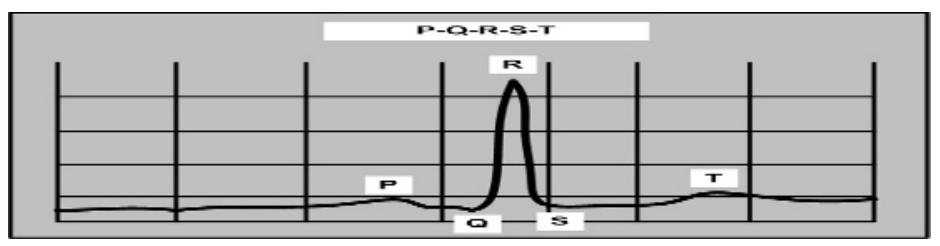

Fig. 5. Pulse rate of heart with $P, Q, R, S$, and $T$ values extracted from the ECG

\subsection{Sensing Context and Home Service Management}

We handled all sensing contexts and control of home service using PDA and PC. Figure 6 shows management screen of all contexts and home service in the ubiquitous intelligent sensing system. (A) and (B) in figure 6 show the manager toolkit that controls the intelligent sensing system by PDA.

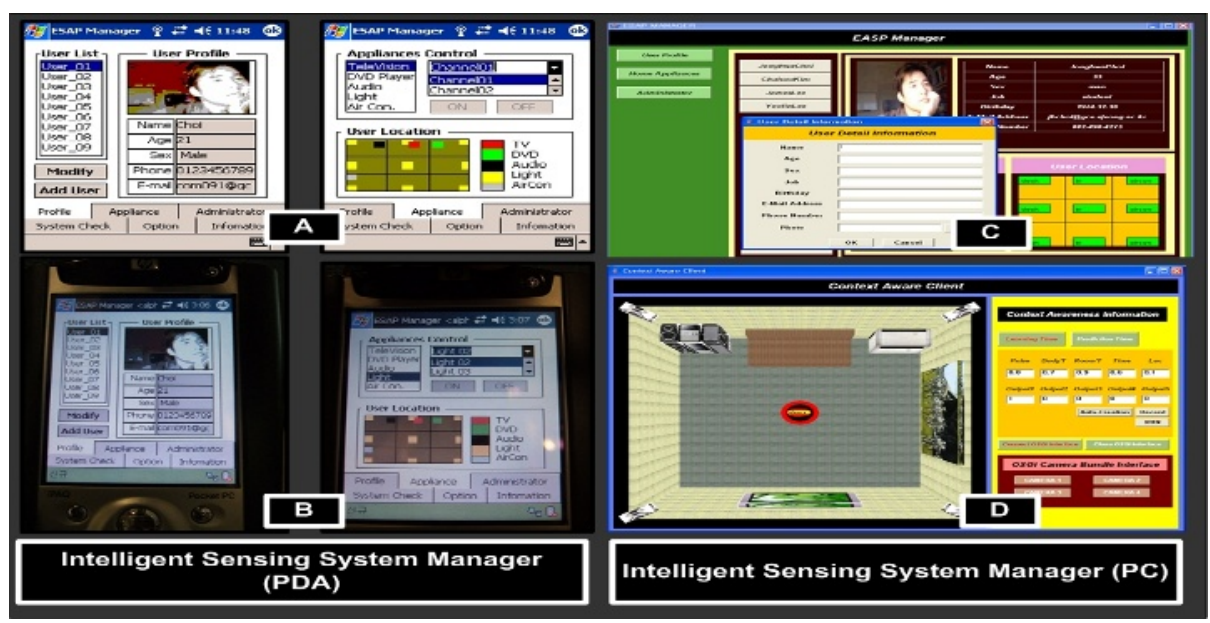

Fig. 6. The manager toolkit for management of the ubiquitous intelligent sensing system

(D) in figure 6 expresses the home's structure and sensing contexts in three dimensions according to the HHIML's structure, which was created from the ubiquitous intelligent sensing system.

\section{Experiments and Evaluations}

The ubiquitous intelligent sensing system provides an automatic home service that is predicted by the pattern recognition algorithm. All contexts were applied as LSVM's features and association rule's input data. The ubiquitous intelligent sensing system provides predicted home services if prediction results of two pattern recognition algorithms (the SAPR and the data miner) are the same. Figure 7 shows a distribution chart of sensing contexts that is presented by the data miner. 


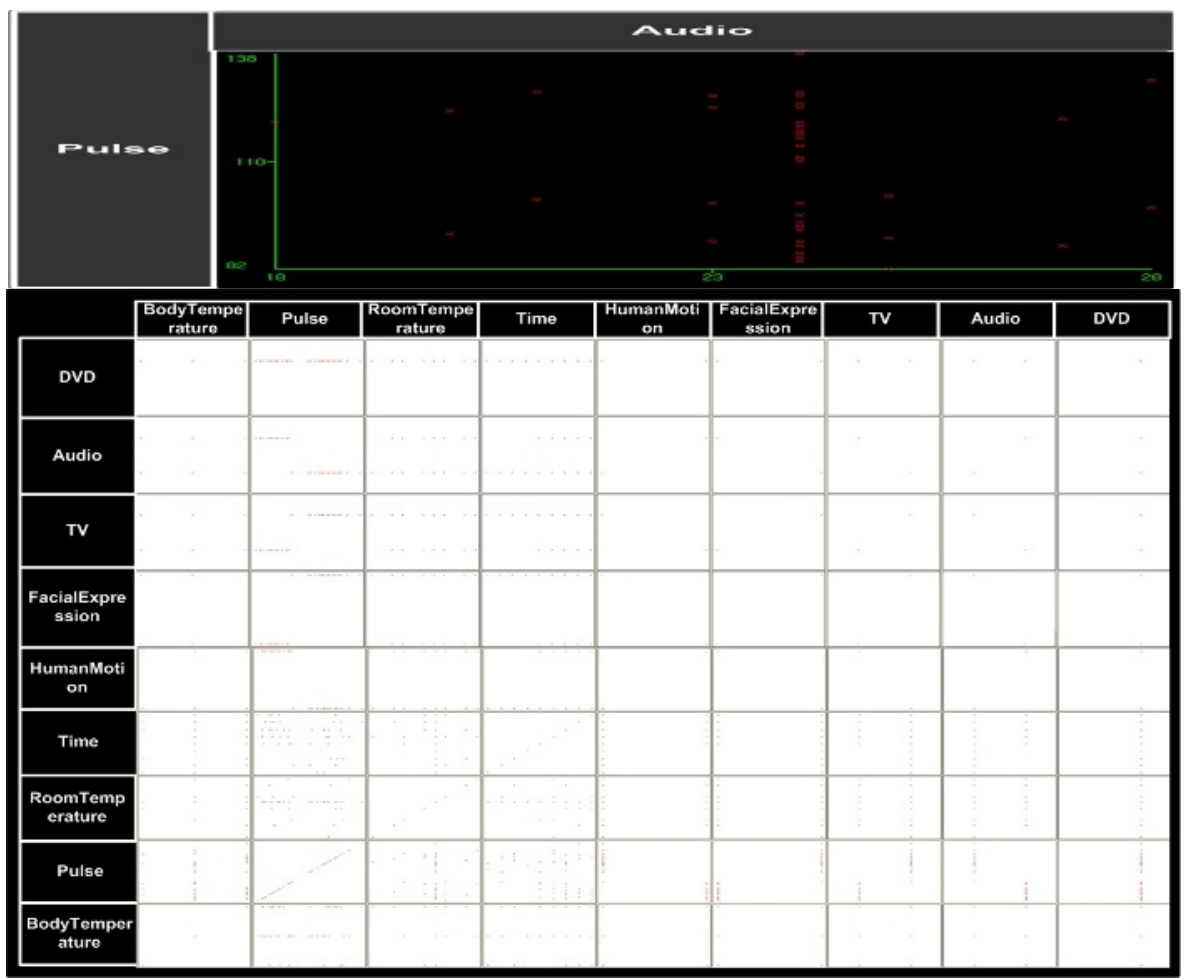

Fig. 7. Distribution chart of sensing contexts that is presented by the data miner

Table 1 shows the performance of the prediction component that is created by the LSVM and the association rules.

Table. 1. The performance of the prediction component that is created by the LSVM and the association rules

\begin{tabular}{|c|c|l|l|l|l|}
\hline & $\begin{array}{l}\text { Number of } \\
\text { Service } \\
\begin{array}{l}\text { Prediction } \\
\text { by the } \\
\text { SAPA }\end{array}\end{array}$ & $\begin{array}{l}\text { Number of } \\
\text { Service } \\
\text { Prediction } \\
\text { by the } \\
\text { Association } \\
\text { Rules }\end{array}$ & $\begin{array}{l}\text { Number of } \\
\text { Service } \\
\text { Prediction } \\
\text { by Integration } \\
\text { Methods }\end{array}$ & $\begin{array}{l}\text { Number of } \\
\text { Rejected } \\
\text { Service by } \\
\text { Human }\end{array}$ & $\begin{array}{l}\text { Precision } \\
\text { on test set }\end{array}$ \\
\hline TV & 123 & 148 & 76 & 21 & $72.3 \%$ \\
\hline DVD & 156 & 111 & 90 & 24 & $73.3 \%$ \\
\hline Audio & 211 & 154 & 107 & 31 & $71.0 \%$ \\
\hline
\end{tabular}

Home service prediction of the ubiquitous intelligent sensing system showed an average $72.2 \%$. We are progressing with experiments of additional sensing contexts to increase the system's performance. Also, we are testing how the performance of the system change by changing the importance of different feature sets. 
The ECG is a sensing context that is applied to measure the human's stress. The ECG wave means a healthy state if it presents big oscillation within the standard scope. Otherwise, it means that the autonomic nervous system's ability to adapt to stress decreases. (a) shows a normal human's ECG wave, and (b) shows human's ECG wave with stress. If a human is suffering from stress, the ubiquitous intelligent sensing system provides home service (soft music and a faint light) that comforts to human and reduces stress.

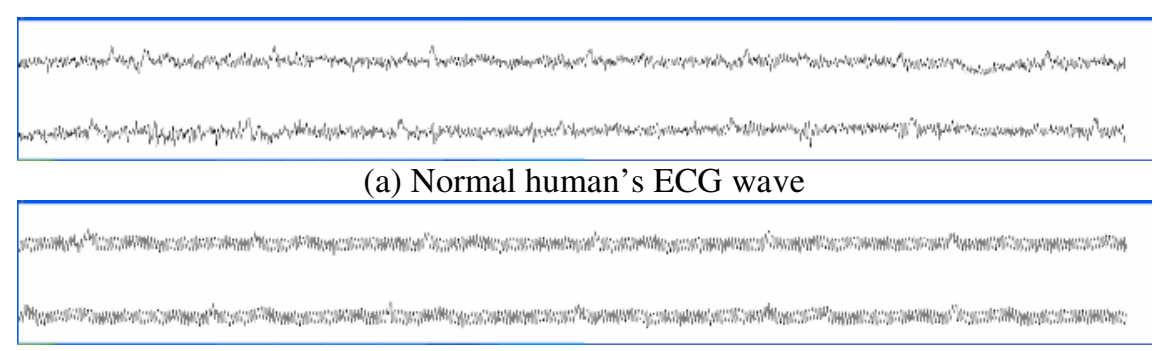

(b) Human's ECG wave with stress

Fig. 8. ECG signals that is extracted from the ECG sensor device

\section{Conclusions}

We presented the ubiquitous intelligent sensing system for a smart home in this paper. A smart home is intelligent space that studies pattern of home contexts that is acquired in a home, and provides an automatic home service for the human. We implemented a smart home using the ubiquitous intelligent sensing system. The ubiquitous intelligent sensing system acquires seven sensing contexts from four sensor devices. This paper uses association rules of data mining and linear support machine to analyze context patterns of seven contexts. Also, we analyze stress rate of human through the HRV pattern of the ECG. If human is suffering from stress, the ubiquitous intelligent sensing system provides home service that reduces human's stress. In this paper, we present the architecture of ubiquitous intelligent sensing system, explains algorithms of components that compose the ubiquitous intelligent sensing system. We present the management toolkit to control the ubiquitous intelligent sensing system, and shows pictures that is executed in the ubiquitous intelligent sensing system.

\section{References}

1. Petriu. E.M, Georganas. N.D, Petriu. D.C, Makrakis. D, Groza. V.Z.: Sensor-based information appliances. Instrumentation \& Measurement Magazine. IEEE Volume 3. Issue 4. (2000) 31 - 35

2. J. Birnbaum.: Pervasive information systems. Communications of the ACM. vol 40. no 2. (1997) 40-41

3. M. L. Dertouzos.: The future of computing. Scientific American. vol 52. (1999) 52-55 
4. M. Weiser.: Some computer science issues in ubiquitous computing. Commun ACM. vol 36. no 7. (1993) 75-84

5. Easy Living. http://research.microsoft.com/easyliving/

6. MavHome. http://mavhome.uta.edu/

7. D.J. Cook, M. Youngblood, E. Heierman, K. Gopalratnam, S. Rao, A. Litvin, and F. Khawaja.: Mavhome: An agent based smart home. Proceedings of the IEEE International Conference on Pervasive Computing and Communications. (2003) 521-524

8. Perry. M, Dowdall. A, Lines. L, Hone. K.: Multimodal and ubiquitous computing systems: supporting independent-living older users. Information Technology in Biomedicine. IEEE Transactions on. Volume 8. Issue 3. (2004) $258-270$

9. Barger. T.S, Brown. D.E, Alwan. M.: Health-Status monitoring through analysis of behavioral patterns. Systems, Man and Cybernetics, Part A, IEEE Transactions on. Volume 35. Issue 1. (2005) $22-27$

10. Mihailidis. A, Carmichael. B, Boger. J.: The use of computer vision in an intelligent environment to support aging-in-place, safety, and independence in the home. Information Technology in Biomedicine. IEEE Transactions on. Volume 8. Issue 3. (2004) 238 - 247

11. Adomavicius. G, Tuzhilin. A.: Using data mining methods to build customer profiles. Computer. Volume 34. Issue 2. (2001) 74 - 82

12. Kehagias. A, Petridis. V.: Predictive modular fuzzy systems for intelligent sensing. Systems, Man, and Cybernetics, IEEE International Conference on. Volume 4. (1996) 2816 - 2821

13. World Wide Web Consortium (W3C). Extensible Markup Language (XML) 1.0. Available: http://www.w3c.org/TR/REC-xml. (1998)

14. Gunhee Kim, Dongkyoo Shin, Dongil Shin.: Design of a middleware and HIML(Human Interaction Markup Language) for context aware services in ubiquitous computing environment. Lecture Notes in computer science. Vol 3207. (2004) 682-691

15. Charles, D.: The expression of the emotions in man and animals. Electronic Text Center. University of Virginia Library

16. Task Force of the European Society of Cardiology and the North American Society of Pacing and Electrophysiology.: Heart Rate Variability standards of measurement. physiological interpretation, and clinical use. Cir. 93. (1996) 1043-1065

17. Lombardi $\mathrm{F}$ and Sandrone G.: Heart rate variability and sympatho-vagal interaction after myocardial infarction. In: Malik M and Camm AJ, Heart Rate Variability. Armonk NY: Futura Publishing Company, Inc. (1995) 222-234 